\title{
Machine Learning of Higher Order Programs*
}

\author{
Ganesh Baliga $^{\dagger} \quad$ John Case ${ }^{\ddagger} \quad$ Sanjay Jain ${ }^{\S} \quad$ Mandayam Suraj $₫$
}

March 11, 2007

\begin{abstract}
A generator program for a computable function (by definition) generates an infinite sequence of programs all but finitely many of which compute that function. Machine learning of generator programs for computable functions is studied. To partially motivate these studies, it is shown that, in some cases, interesting global properties for computable functions can be proved from suitable generator programs which can not be proved from any ordinary programs for them. The power (for variants of various learning criteria from the literature) of learning generator programs is compared with the power of learning ordinary programs. The learning power in these cases is also compared to that of learning limiting programs, i.e., programs allowed finitely many mind changes about their correct outputs.
\end{abstract}

\section{Preliminaries}

\section{$1.1 \quad$ Notation}

Any unexplained recursion theoretic notation is from [Rog67]. $N$ denotes the set of natural numbers, $\{0,1,2,3, \ldots\}$. Unless otherwise specified, $b, e, i, j, k, l, m, n, p, r, s, t, w, x, y, z$, with or without decorations ${ }^{1}$,

*Preliminary version of this paper appeared in the Proceedings of the Second Symposium on Logical Foundations of Computer Science, Tver, Russia, July, 1992.

${ }^{\dagger}$ Department of Computer and Information Sciences, University of Delaware, Newark, DE 19716. Email: baliga@cis.udel.edu. ${ }^{\ddagger}$ Department of Computer and Information Sciences, University of Delaware, Newark, DE 19716. Email: case@cis.udel.edu.

§Institute of Systems Science, National University of Singapore, Singapore 0511. Email: sanjay@iss.nus.sg.

๑Department of Computer and Information Sciences, University of Delaware, Newark, DE 19716. Email: suraj@cis.udel.edu.

${ }^{1}$ Decorations are subscripts, superscripts and the like. 
range over $N$. * denotes a non-member of $N$ and is assumed to satisfy $(\forall n)[n<*<\infty]$. $a$ with or without decorations, ranges over $N \cup\{*\}$. $\emptyset$ denotes the empty set. $\subseteq$ denotes subset. $\subset$ denotes proper subset. For $S$, a subset of $N, \operatorname{card}(S)$ denotes the cardinality of $S$. $\uparrow$ denotes undefined. $\max (\cdot), \min (\cdot)$ denote the maximum and minimum of a set, respectively, where $\max (\emptyset)=0$ and $\min (\emptyset)=\uparrow$.

$\eta$ and $\theta$ range over partial functions with arguments and values from $N . \eta(x) \downarrow$ denotes that $\eta(x)$ is defined; $\eta(x) \uparrow$ denotes that $\eta(x)$ is undefined.

$f, g, h, L, q$ with or without decorations range over total functions with arguments and values from $N$. For $n \in N$ and partial functions $\eta$ and $\theta, \eta={ }^{n} \theta$ means that $\operatorname{card}(\{x \mid \eta(x) \neq \theta(x)\}) \leq n ; \eta={ }^{*} \theta$ means that $\operatorname{card}(\{x \mid \eta(x) \neq \theta(x)\})$ is finite. domain $(\eta)$ and range $(\eta)$ denote the domain and range of the function $\eta$, respectively.

We say that $\eta$ is monotone $\stackrel{\text { def }}{\Leftrightarrow}(\forall x, y \mid x<y)[\eta(x) \downarrow<\eta(y) \downarrow]$. Thus $\eta$ is monotone iff $\eta$ is a strictly increasing total function.

$\langle i, j\rangle$ stands for an arbitrary, computable, one-to-one encoding of all pairs of natural numbers onto $N$ [Rog67]. Similarly we can define $\langle\cdot, \ldots, \cdot\rangle$ for encoding multiple natural numbers onto $N$.

$\varphi$ denotes a fixed acceptable programming system for the partial computable functions: $N \rightarrow N[\operatorname{Rog} 58$, Rog67, MY78]. $\varphi_{i}$ denotes the partial computable function computed by program $i$ in the $\varphi$-system. $\Phi$ denotes an arbitrary Blum complexity measure [Blu67, HU79] for the $\varphi$-system. $K$ denotes $\left\{p \mid \varphi_{p}(p) \downarrow\right\} . K$ is a standard r.e. set with $\bar{K}$, the complement of $K$, being constructively non-r.e. [Rog67].

The set of all total computable functions of one variable is denoted by $\mathcal{R}$. $\mathcal{C}$, with or without decorations, ranges over subsets of $\mathcal{R}$. For computable $f, \operatorname{MinProg}(f)$ denotes $\min \left(\left\{i \mid \varphi_{i}=f\right\}\right)$.

We sometimes consider partial computable functions with multiple arguments in the $\varphi$ system. In such cases we implicitly assume that a $\langle\cdot, \ldots, \cdot\rangle$ is used to code the arguments, so, for example, $\varphi_{i}(x, y)$ stands for $\varphi_{i}(\langle x, y\rangle)$.

The quantifier ' $\forall$ ' essentially from [Blu67], means 'for all but finitely many'. ' $\exists$ !' means 'there exists an unique'.

\subsection{Fundamental Function Inference Paradigms}

A Learning Machine (LM) [Gol67] is an algorithmic device which takes as its input a set of data given one element at a time, and which from time to time, as it is receiving its input, outputs programs. LMs have 
been used in the study of machine learning or inductive inference of programs for computable functions as well as algorithmic learning of grammars for languages [BB75, CS83, Che81, Ful85, Gol67, OSW86, Wie78, AS83, KW80, Cas86]. ${ }^{2}$

M, with or without decorations, ranges over the class of LMs. For the learning of a computable function $f$ by an LM, $\mathbf{M}$, the graph of $f$ is fed to $\mathbf{M}$ in any order. Without loss of generality [BB75, CS83], we will assume that $\mathbf{M}$ is fed the graph of $f$ in the sequence $(0, f(0)),(1, f(1)),(2, f(2)), \ldots$. For all computable functions $f, f[n]$ denotes the finite initial segment $((0, f(0)),(1, f(1)), \ldots,(n-1, f(n-1)))$. Let INIT $=$ $\{f[n] \mid f \in \mathcal{R} \wedge n \in N\} . \sigma$, with or without decorations, ranges over INIT. $\mathbf{M}(\sigma)$ is the last output of $\mathbf{M}$ by the time it receives all of $\sigma$. For the learning criteria discussed in this paper, we can and will assume, without loss of generality, that $\mathbf{M}(\sigma)$ is always defined. We say that $\mathbf{M}(f)$ converges to $i$ (written: $\mathbf{M}(f) \downarrow=i)$ iff $(\stackrel{\infty}{\forall} n)[\mathbf{M}(f[n])=i] ; \mathbf{M}(f)$ is undefined if no such $i$ exists.

Recall, that according to our convention $a \in N \cup\{*\}$.

Definition 1 [Gol67, BB75, CS83]

(a) $\mathbf{M} \mathbf{E x}^{a}$-identifies a computable function $f$ (written: $f \in \mathbf{E x}^{a}(\mathbf{M})$ ) iff both $\mathbf{M}(f) \downarrow$ and $\varphi_{\mathbf{M}(f)}={ }^{a} f$.

(b) $\mathbf{E x}^{a}=\left\{\mathcal{C} \subseteq \mathcal{R} \mid(\exists \mathbf{M})\left[\mathcal{C} \subseteq \mathbf{E x}^{a}(\mathbf{M})\right]\right\}$

Case and Smith [CS83] introduced another infinite hierarchy of learning criteria which we describe below. "Bc" stands for behaviorally correct. Barzdin [Bar74] essentially introduced the notion Bc ${ }^{0}$.

\section{Definition 2 [CS83]}

(a) $\mathbf{M} \mathbf{B} \mathbf{c}^{a}$-identifies a computable function $f$ (written: $\left.f \in \mathbf{B c}^{a}(\mathbf{M})\right)$ iff $(\stackrel{\infty}{\forall} n)\left[\varphi_{\mathbf{M}(f[n])}={ }^{a} f\right]$.

(b) $\mathbf{B c}^{a}=\left\{\mathcal{C} \subseteq \mathcal{R} \mid(\exists \mathbf{M})\left[\mathcal{C} \subseteq \mathbf{B c}^{a}(\mathbf{M})\right]\right\}$.

We usually write $\mathbf{E x}$ for $\mathbf{E} \mathbf{x}^{0}$ and $\mathbf{B c}$ for $\mathbf{B} \mathbf{c}^{0}$. Theorem 3 just below states some of the basic hierarchy results about the $\mathbf{E} \mathbf{x}^{a}$ and $\mathbf{B} \mathbf{c}^{a}$ classes.

Theorem 3 For all $n$,

(a) $\mathbf{E x}^{n} \subset \mathbf{E} \mathbf{x}^{n+1}$

\footnotetext{
${ }^{2}$ We have not yet investigated language learning analogs of our results.
} 
(b) $\bigcup_{n \in N} \mathbf{E x}^{n} \subset \mathbf{E x}$,

(c) $\mathbf{E x}^{*} \subset \mathbf{B c}$,

(d) $\mathbf{B c}^{n} \subset \mathbf{B c}^{n+1}$,

(e) $\bigcup_{n \in N} \mathbf{B c}^{n} \subset \mathbf{B c}^{*}$, and

(f) $\mathcal{R} \in \mathbf{B} \mathbf{c}^{*}$.

Parts (a), (b), (d), and (e) are due to Case and Smith [CS83]. John Steel first observed that $\mathbf{E x}^{*} \subseteq \mathbf{B c}$ and the diagonalization in part (c) is due to Harrington and Case [CS83]. Part (f) is due to Harrington [CS83]. Blum and Blum [BB75] first showed that Ex $\subset$ Ex* $\mathbf{x}^{*}$ Barzdin [Bar74] first showed that Ex $\subset$ Bc.

\section{$2 \quad$ Higher Order Programs}

\subsection{Definition and Motivation of Higher Order Programs}

We consider two kinds of higher order programs: limiting programs (from [CJS92]) and generator programs (introduced in the present paper).

First we discuss limiting programs.

For each $i$, consider the following corresponding procedure for "computing" a (partial) function $\varphi_{i}^{\star}$.

On input $x$

for $t=0$ to $\infty$

Start a new clone of $\varphi$-program $i$ running on input $(x, t)$

endfor

It is to be understood that

(a) each iterate of the for-loop finishes since it merely starts a process running and

(b) in some iterates of the for-loop the process started may itself never converge.

$\varphi_{i}^{\star}(x) \stackrel{\text { def }}{=}$ the unique $y$ (if any) eventually output by all but finitely many of the clones of $\varphi$-program $i$ in the for-loop above. Equivalently, $\varphi_{i}^{\star}(x) \stackrel{\text { def }}{=} \lim _{t \rightarrow \infty} \varphi_{i}(x, t)$. 
We shall refer to $i$ as Lim-program $i$ (in the $\varphi^{\star}$-system) when we are thinking of $i$ as encoding the for-loop above rather than as encoding $\varphi$-program $i$.

Intuitively, Lim-program $i$ (in the $\varphi^{\star}$-system) is a procedure, which on an input for which it has an output, is allowed to change its mind finitely many times about that output (or even about whether to output at all). N.B. there may be no algorithm for signaling when a Lim-program has stopped changing its mind about its output.

The partial functions which are the limit of some total computable function are well known to be characterized as exactly the partial functions computable relative to an oracle for the halting problem [Sho59, Put65, Gol65, Sho71, Soa87]. ${ }^{3}$ This result and its relativizations were first noticed and used by Post [Sha71] and have been employed (sometimes with rediscovery) many times. [LMF76] studied acceptable programming systems for partial functions computable relative to oracles. Many of the results of this paper about Lim-programs would hold also for programs in acceptable oracular programming systems with oracle for the halting problem attached, but we will present our Lim-program results directly about systems such as $\varphi^{\star}$.

In the present paper, as in [CJS92], we shall be especially interested in Lim-programs (from the $\varphi_{i}^{\star}$ system) which happen to compute partial computable functions. The learning of Lim-programs for computable functions is compared to the learning of ordinary $\varphi$-programs in [CJS92].

The reader might think that Lim-programs for computable functions $f$ are not particularly useful, but with such programs one can discover values for $f$ eventually. However, one may not know when one has found those values, and it is easy to argue that "eventually" is too long to wait.

Actually, Lim-programs can be quite useful.

In physics it is sometimes easier to infer a global property of a phenomenon than it is to make more detailed predictions about observations; for example, Kepler's Law that the planets orbit in ellipses is easier to derive than equations of motion of planets. In Section 2.2 we state a result, Theorem 5 , extending a result from [CJS92], that it is, in some cases, possible to prove global properties of a computable function from a suitable Lim-program for it when it is not possible to prove these properties from any of the ordinary $(\varphi)$ programs for it.

Next we discuss generator programs.

\footnotetext{
${ }^{3}$ The class of partial functions which are the limit of some partial computable function, i.e., $\left\{\varphi_{i}^{\star} \mid i \in N\right\}$, is a larger class than the class of partial functions computable in the halting problem.
} 
Informally, a $\varphi$-program $p$ is a 0 -generator program for $f$ just in case $\varphi_{p}$ is total and all but finitely many of the programs $\varphi_{p}(0), \varphi_{p}(1), \varphi_{p}(2), \ldots$ compute $f .{ }^{4}$

Definition 4 We say that $\varphi$-program $p$ is an a-generator for $f$ iff

(a) $\varphi_{p} \in \mathcal{R}$ and

(b) $(\stackrel{\infty}{\forall} x)\left[\varphi_{\varphi_{p}(x)}={ }^{a} f\right]$.

Our remarks above about the possible usefulness of Lim-programs can be applied mutatis mutandis to $a$-generators. An a-program for $f$ is a program $p$ such that $\varphi_{p}={ }^{a} f$. In the next section (Section 2.2 ) we present results to the effect that, for $a=0,1$, respectively, it is, in some cases, possible to prove global properties of a computable function from a suitable $a$-generator program for it when it is not possible to prove these properties from any of the ordinary $b$-programs for it, for $b=0$, , respectively. We also present such a result comparing Lim-programs with 0-generator programs.

\subsection{Further Motivation}

We next provide the preliminaries for obtaining the provability results as advertised above in the previous section (Section 2.1).

We present our results for extensions of first order arithmetic. Regarding expressing propositions in first order arithmetic, we shall proceed informally. If $\mathrm{E}$ is an expression such as ' $\Phi_{i} \leq t$ ', or ' $\varphi_{i}$ is monotone', we shall write $\ll E \gg$ to denote a naturally corresponding, fixed standard wff of first order arithmetic [Men86] which (semantically) expresses E. We need and assume that

if $E^{\prime}$ is obtained from $E$ by changing some numerical values, then $\ll E^{\prime} \gg$ can be algorithmically obtained from those changed numerical values and $\ll E \gg$.

It is understood that, if $E$ contains references to partial functions, such as $\varphi$ and $\Phi$, then in $\ll E \gg$ these are, in effect, named by standard programs for them. It is well known that wffs extensionally equivalent (with respect to standard models) may not be intensionally or provably equivalent [Fef60]. In what follows, when we use the $\ll E \gg$ notation, it will always be for propositions that are easily seen to be (semantically) expressible in first order arithmetic. ${ }^{5}$ ' $\vdash$ ' denotes the provability relation.

\footnotetext{
${ }^{4}$ It is without loss of generality for our learning criteria introduced in Section 3 below that we require $\varphi_{p}$ be total.

${ }^{5}$ This informal discussion of provability and expressibility is based on Section 4.3 of [RC92].
} 
The following theorem extends slightly a theorem from [CJS92] and motivates the usefulness of Limprograms over ordinary $\varphi$-programs. The furthermore clause is new. Grigori Schwarz suggested to us the problem of whether it could be added.

Theorem 5 Suppose $\mathbf{T}$ is an axiomatizable (i.e., r.e. [Cra53]) first order theory which extends Peano Arithmetic [Men86] and in which one can not prove anything false about monotonicity of (partial) computable functions computed by programs in $\varphi$. Then there exist $f \in \mathcal{R}$ and e such that $\varphi_{e}^{\star}=f$ and $f$ is monotone, yet

(a) $\left(\forall i \mid \varphi_{i}=f\right)\left[\mathbf{T} \nvdash \ll \varphi_{i}\right.$ is monotone $\left.\gg\right]$ and

(b) $\left[\mathbf{T} \vdash \ll \varphi_{e}^{\star}\right.$ is monotone $]$.

Furthermore, $\left[\mathbf{T} \vdash \ll \varphi_{e}^{\star}\right.$ is computable $\left.\gg\right]$.

The proof of Theorem 5 can be obtained by a simple modification of its predecessor in [CJS92]. The proof of Theorem 11 below can be similarly obtained. We omit the details for both. An anonymous referee nicely pointed out that, if we replace the global property of 'monotone' in Theorems 5 and 11 by 'total' and note that (i) there is an r.e. set of Lim-programs for $\mathcal{R}$ and (ii) there is neither an r.e. set of (ordinary) programs nor an r.e. set of 0 -generators for $\mathcal{R}$, then these modified theorems involve the well studied provably recursive functions [Kre51, Kre58, Fis65, Rog57, Ros84] and quite easily follow. We originally chose to work with monotonicity, rather than totality, since it is about the global shape of a curve and, hence, a better analog of the elliptical shape of orbits.

Next we present the advertised two theorems (Theorem 6 and 10) motivating the usefulness of generator programs over ordinary programs. The range containment property featured in Theorem 6 is a somewhat technical global property for computable functions. Theorem 10 deals with a variant of the monotonicity property of functions, and this property is clearly an interesting global property.

Theorem 6 Suppose $\mathbf{T}$ is an axiomatizable (i.e., r.e.) first order theory which extends Peano Arithmetic and in which one can not prove anything false about the containment of ranges of (partial) computable functions (computed by programs in $\varphi$ ) in $\bar{K}$. Then there exist $f \in \mathcal{R}$ and $p_{0}$ such that $\varphi_{p_{0}}$ is total, $p_{0}$ is a 0 -generator for $f$, and range $(f) \subseteq \bar{K}$, yet

(a) $\left(\forall i \mid \varphi_{i}=f\right)\left[\mathbf{T} \nvdash \ll \operatorname{range}\left(\varphi_{i}\right) \subseteq \bar{K} \gg\right]$ and 
(b) $\mathbf{T} \vdash \ll(\forall t)\left[\operatorname{range}\left(\varphi_{\varphi_{p_{0}}}(t) \subseteq \bar{K}\right] \gg\right.$.

Furthermore, $\mathbf{T} \vdash \ll\left[(\forall t)\left[\varphi_{\varphi_{p_{0}}(t)} \in \mathcal{R}\right] \wedge(\stackrel{\infty}{\forall} t)\left[\varphi_{\varphi_{p_{0}}(t)}=\varphi_{\varphi_{p_{0}}(t+1)}\right]\right] \gg$.

Proof. Suppose the hypotheses. Fix an automatic theorem prover for T. In what follows, any reference to proving something in $\mathbf{T}$ within so many steps refers to steps in the execution of this automatic theorem prover.

Note that, since $\bar{K}$ is not r.e., $\left\{x \mid x \in \bar{K} \wedge\left(\forall i \mid x \in \operatorname{range}\left(\varphi_{i}\right)\right)\left[\mathbf{T} \nvdash \ll \operatorname{range}\left(\varphi_{i}\right) \subseteq \bar{K} \gg\right]\right\}$ is not empty; hence, $z_{0} \stackrel{\text { def }}{=} \min \left(\left\{x \mid x \in \bar{K} \wedge\left(\forall i \mid x \in \operatorname{range}\left(\varphi_{i}\right)\right)\left[\mathbf{T} \nvdash \ll \operatorname{range}\left(\varphi_{i}\right) \subseteq \bar{K} \gg\right]\right\}\right)$ is in $\bar{K}$.

Let $z_{1}$ be such that $\mathbf{T} \vdash \ll z_{1} \in \bar{K} \gg$.

For each $t$, let $S_{t}=\left\{\varphi_{j}(y) \mid j \leq t \wedge y \leq t \wedge \Phi_{j}(y) \leq t\right.$ and $\mathbf{T} \vdash \ll \operatorname{range}\left(\varphi_{j}\right) \subseteq \bar{K} \gg$ in $\leq t$ steps $\}$ and let $T_{t}=\left\{x \leq t \mid \Phi_{x}(x) \leq t\right\}$. Note that, for all $t, S_{t} \subseteq \bar{K}$ and $T_{t} \subseteq K$. Moreover, canonical indices [Rog67] for the finite sets $S_{t}$ and $T_{t}$ can be found algorithmically from $t$.

Let $h$, computable, be such that for all $x, y, \varphi_{h(x)}(y)=x$ (by Kleene's s-m-n theorem [Rog67] such an $h$ exists).

Let $p_{0}$ be such that,

$$
\varphi_{p_{0}}(t)= \begin{cases}h\left(\min \left(\overline{S_{t} \cup T_{t}}\right)\right), & \text { if } S_{t} \cap T_{t}=\emptyset ; \\ h\left(z_{1}\right), & \text { otherwise. }\end{cases}
$$

A simple analysis shows that $z_{0}=\lim _{t \rightarrow \infty}\left[\min \left(\overline{S_{t} \cup T_{t}}\right)\right]$. Let $f=\lambda x \cdot z_{0}$. Note that $\left(\stackrel{\infty}{\forall t)}\left[\varphi_{\varphi_{p_{0}}(t)}=f\right]\right.$. Clearly, $\left(\forall i \mid \varphi_{i}=f\right)\left[\mathbf{T} \forall \ll \operatorname{range}\left(\varphi_{i}\right) \subseteq \bar{K} \gg\right]$ (by the definition of $z_{0}$ ). Since, in Peano Arithmetic, it can be proved that $\ll\left[(\forall t)\left[S_{t} \cap T_{t}=\emptyset\right] \Rightarrow\left[\lim _{t \rightarrow \infty}\left(\overline{S_{t} \cup T_{t}}\right) \neq \emptyset\right]\right] \gg$ and also that $\ll\left[(\exists t)\left[S_{t} \cap T_{t} \neq\right.\right.$ $\left.\emptyset] \Rightarrow(\stackrel{\otimes}{\forall} t)\left[S_{t} \cap T_{t} \neq \emptyset\right]\right] \gg$, it is easy to show in Peano Arithmetic that $\ll\left[(\stackrel{\infty}{\forall} t)\left[\operatorname{range}\left(\varphi_{\varphi_{p_{0}}(t)}\right) \subseteq \bar{K}\right]\right] \gg$.

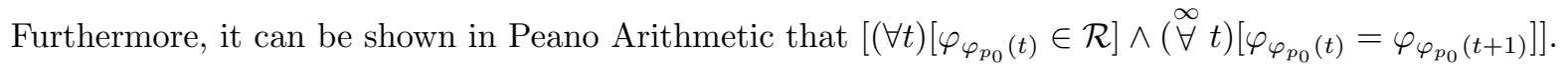

We will now consider the monotonicity result for the 1-generator programs. First we present a few definitions.

Definition 7 Let $a \in N \cup\{*\}$. We say that $p$ is an a-nice_generator for $f \in \mathcal{R}$, iff $\varphi_{p} \in \mathcal{R}$, and, either

(a) $(\stackrel{\forall}{\forall})\left[\varphi_{\varphi_{p}(n)}=f\right]$ or

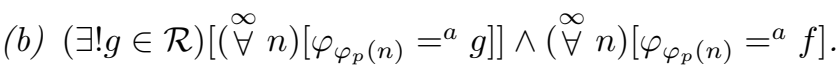

Definition 8 Let $a \in N \cup\{*\}$. We say that $p$ is a-nice_gen_monotone iff $(\exists f \in \mathcal{R} \mid f$ is monotone $)[p$ is a-nice_generator for $f]$. 
Definition 9 Let $a \in N \cup\{*\}$. We say that $i$ is a-monotone iff $(\exists f \in \mathcal{R} \mid f$ is monotone $)\left[\varphi_{i}={ }^{a} f\right]$.

Theorem 10 Suppose $\mathbf{T}$ is an axiomatizable (i.e., r.e.) first order theory which extends Peano Arithmetic, and in which one can not prove anything false of the form ' $i$ is $*$-monotone'. Then there exist $f \in \mathcal{R}$ and $p_{0}$ such that $\varphi_{p_{0}}$ is total, $p_{0}$ is 1-nice_generator for $f$, and $f$ is monotone, yet

(a) $\left(\forall i \mid \varphi_{i}={ }^{*} f\right)[\mathbf{T} \nvdash \ll i$ is $*$-monotone $\gg]$ and

(b) $\left[\mathbf{T} \vdash \ll p_{0}\right.$ is 1-nice_gen_monotone $\left.\gg\right]$.

Proof. Suppose the hypotheses. Fix an automatic theorem prover for T. As in the proof of Theorem 6 above, in what follows any reference to proving something in $\mathbf{T}$ within so many steps refers to steps in the execution of this automatic theorem prover.

By the operator recursion theorem [Cas74] there exists a $p_{0}$, such that the (partial) functions $\varphi_{\varphi_{p_{0}}(\cdot)}$ may be described as follows. The (partial) functions $\varphi_{\varphi_{p_{0}}(\cdot)}$ are described in stages.

Let $\varphi_{\varphi_{p_{0}}(0)}(0)=0 . x_{s}$ denotes the least input on which $\varphi_{\varphi_{p_{0}}(0)}$ has not been defined before stage $s . l_{s}$ denotes the least number such that, $\varphi_{\varphi_{p_{0}}\left(l_{s}\right)}$ has not been defined on any input before the start of stage $s$. Thus $x_{0}=1$ and $l_{0}=1$. Go to stage 0 .

Begin stage $s$

1. For all $x<x_{s}$, let $\varphi_{\varphi_{p_{0}}\left(l_{s}\right)}(x)=\varphi_{\varphi_{p_{0}}(0)}(x)$.

2. Let $r_{s}=l_{s}$ and $y=x_{s}-1$.

3. Let $P=\{j \leq s \mid \mathbf{T} \vdash \ll j$ is $*$-monotone $\gg$ in $\leq s$ steps $\}$.

4. repeat

$4.1 r_{s}=r_{s}+1 ; y=y+1$.

4.2 For $x<y$, let $\varphi_{\varphi_{p_{0}}\left(r_{s}\right)}(x)=\varphi_{\varphi_{p_{0}}\left(l_{s}\right)}(x)$.

For $x \geq y$, whenever $\varphi_{\varphi_{p_{0}}\left(l_{s}\right)}(x)$ gets defined, let $\varphi_{\varphi_{p_{0}}\left(r_{s}\right)}(x)=\varphi_{\varphi_{p_{0}}\left(l_{s}\right)}(x)$ (i.e. $\varphi_{\varphi_{p_{0}}\left(r_{s}\right)}$ "follows" $\varphi_{\varphi_{p_{0}}\left(l_{s}\right)}$ from now on. Note that because of this step $\left.\varphi_{\varphi_{p_{0}}\left(r_{s}\right)}=\varphi_{\varphi_{p_{0}}\left(l_{s}\right)}\right)$.

4.3 Let $\varphi_{\varphi_{p_{0}}\left(l_{s}\right)}(y)=\varphi_{\varphi_{p_{0}}\left(l_{s}\right)}(y-1)+\operatorname{card}(P)+2$.

4.4 If there exists an $x, x_{s} \leq x<y$, such that $(\forall j \in P)\left[\Phi_{j}(x) \leq y\right]$, then let $z$ be the least such $x$, and go to step 5 .

\section{forever}


5. For $x \in\left\{x \neq z \mid x_{s} \leq x \leq y\right\}$, let $\varphi_{\varphi_{p_{0}}(0)}(x)=\varphi_{\varphi_{p_{0}}\left(l_{s}\right)}(x)$.

Let $\varphi_{\varphi_{p_{0}}(0)}(z)=w$, where $w=\min \left(\left\{x \mid \varphi_{\varphi_{p_{0}}\left(l_{s}\right)}(z)<x<\varphi_{\varphi_{p_{0}}\left(l_{s}\right)}(z+1)\right\}-\left\{\varphi_{j}(z) \mid j \in P\right\}\right)$.

(Note that $w$ is not undefined since, $\varphi_{\varphi_{p_{0}}\left(l_{s}\right)}(z+1)-\varphi_{\varphi_{p_{0}}\left(l_{s}\right)}(z)=\operatorname{card}(P)+2$.)

For $x>y$, whenever $\varphi_{\varphi_{p_{0}}(0)}(x)$ gets defined, let $\varphi_{\varphi_{p_{0}}\left(l_{s}\right)}(x)=\varphi_{\varphi_{p_{0}}(0)}(x)$ (i.e. $\varphi_{\varphi_{p_{0}}\left(l_{s}\right)}$ "follows" $\varphi_{\varphi_{p_{0}}(0)}$ from now on).

(Note that due to step $5, \varphi_{\varphi_{p_{0}}\left(l_{s}\right)}={ }^{1} \varphi_{\varphi_{p_{0}}(0)}$.)

6. Go to stage $s+1$.

(Note that $x_{s+1}=y+1$ and $\left.l_{s+1}=r_{s}+1\right)$.

End stage $s$.

Now define $f$ as follows.

$$
f= \begin{cases}\varphi_{\varphi_{p_{0}}(0)}, & \text { if infinitely many stages are executed; } \\ \varphi_{\varphi_{p_{0}}\left(l_{s}\right)}, & \text { if stage } s \text { starts but never finishes. }\end{cases}
$$

A simple case analysis shows that,

$f$ is monotone, and

$p_{0}$ is a 1 -nice_generator for $f$, and thus $p_{0}$ is 1 -nice_gen_monotone.

Furthermore, this proof of $p_{0}$ being 1-nice_gen_monotone can be formalized in Peano Arithmetic.

Now, since $\mathbf{T}$ does not prove anything false about $*$-monotonicity of programs, all stages halt. This implies that $f=\varphi_{\varphi_{p_{0}}(0)}$. Thus for all $j$, such that $\mathbf{T} \vdash \ll j$ is $*$-monotone $\gg$, there exist infinitely many $x$ such that, $\varphi_{j}(x) \neq f(x)$ (by the diagonalization at step 5 on input $z$, for each stage $s$ ). The theorem follows.

Next is the promised theorem (Theorem 11) comparing Lim-programs and 0-generator programs.

Theorem 11 Suppose $\mathbf{T}$ is an axiomatizable (i.e., r.e.) first order theory which extends Peano Arithmetic

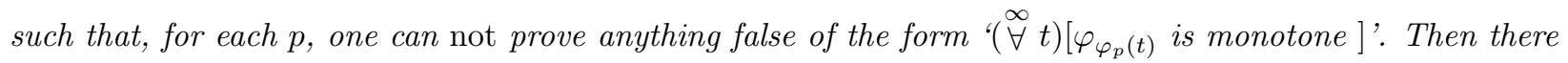
exist $f \in \mathcal{R}$ and $e$ such that $\varphi_{e}^{\star}=f$ and $f$ is monotone, yet

(a) $(\forall p \mid p$ is a 0-generator for $f)\left[\mathbf{T} \nvdash \ll(\forall t)\left[\varphi_{\varphi_{p}(t)}\right.\right.$ is monotone $\left.] \gg\right]$ and

(b) $\left[\mathbf{T} \vdash \ll \varphi_{e}^{\star}\right.$ is monotone $\left.\gg\right]$.

Furthermore, $\left[\mathbf{T} \vdash \ll \varphi_{e}^{\star}\right.$ is computable $\left.\gg\right]$. 


\section{Learning Higher Order Programs}

Definition 12 [CJS92]

(a) A machine $\mathbf{M}, \mathbf{L i m E x}^{a}$-identifies $f$ (written: $f \in \mathbf{L i m E x}^{a}(\mathbf{M})$ ) iff $\mathbf{M}(f) \downarrow=i$ such that $\left[f={ }^{a} \varphi_{i}^{\star}\right]$.

(b) $\operatorname{LimEx} x^{a}=\left\{\mathcal{C} \subseteq \mathcal{R} \mid(\exists \mathbf{M})\left[\mathcal{C} \subseteq \operatorname{LimEx}^{a}(\mathbf{M})\right]\right\}$.

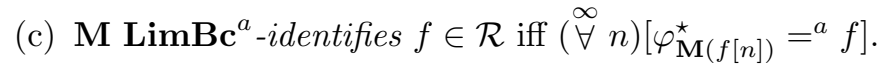

(d) $\operatorname{LimBc}^{a}=\left\{\mathcal{C} \subseteq \mathcal{R} \mid(\exists \mathbf{M})\left[\mathcal{C} \subseteq \operatorname{LimBc}^{a}(\mathbf{M})\right]\right\}$.

We write $\operatorname{LimEx}$ for $\operatorname{LimEx} x^{0}$ and $\operatorname{LimBc}$ for $\operatorname{LimBc}^{0}$. We do not consider $\operatorname{LimBc}^{a}$ further since from [CJS92] $\mathcal{R} \in$ LimBc.

It is shown in [CJS92], for example, that

Theorem 13 For all $a, i$,

(a) $\mathbf{E x}^{a} \subset \mathbf{L i m E x}{ }^{a}$,

(b) $\operatorname{LimEx}-\mathbf{B c}^{i} \neq \emptyset$,

(c) $\mathbf{E x}^{i+1}-\mathbf{L i m E x} \mathbf{x}^{i} \neq \emptyset$,

(d) $\mathbf{E x}^{*}-\bigcup_{i \in N} \operatorname{LimEx^{i}} \neq \emptyset$, and

(e) $\mathbf{B c}-\operatorname{LimEx}^{*} \neq \emptyset$.

\section{Definition 14}

(a) A machine $\mathbf{M}, \mathbf{G e n E x}^{a}$-identifies $f$ (written: $f \in \mathbf{G e n E x}^{a}(\mathbf{M})$ ) iff $\mathbf{M}(f) \downarrow$ to some $a$-generator for $f$.

(b) $\operatorname{GenEx}^{a}=\left\{\mathcal{C} \subseteq \mathcal{R} \mid(\exists \mathbf{M})\left[\mathcal{C} \subseteq \mathbf{G e n E x}^{a}(\mathbf{M})\right]\right\}$.

(c) A machine $\mathbf{M}$, GenBc ${ }^{a}$-identifies $f$ (written: $\left.f \in \mathbf{G e n B c}^{a}(\mathbf{M})\right)$ iff $(\stackrel{\infty}{\forall} n)[\mathbf{M}(f[n])$ is an $a$-generator for $f]$.

(d) $\mathbf{G e n B c}^{a}=\left\{\mathcal{C} \subseteq \mathcal{R} \mid(\exists \mathbf{M})\left[\mathcal{C} \subseteq \operatorname{GenBc}^{a}(\mathbf{M})\right]\right\}$.

We write GenEx for $\mathbf{G e n E x}^{0}$ and $\mathbf{G e n B c}$ for $\mathbf{G e n B c}^{0}$. The terminology in Definition 14 just above should not be confused with that in [CL82]. 


\subsection{Results}

The next theorem (Theorem 15) leaves open the questions of whether some containment relations are proper.

These questions are settled by the end of the paper.

Theorem 15 For all a,

(a) $\mathbf{E x}^{a} \subseteq \mathbf{G e n E x}^{a}$

(b) $\operatorname{GenEx}^{a} \subseteq \mathbf{B c}^{a}$,

(c) GenEx $=\mathbf{E x}$, and

(d) $\mathcal{R} \in$ GenBc.

ProOF.

(a), (b) Easy to prove.

(c) Given $\mathbf{M}$, we will construct $\mathbf{M}^{\prime}$ such that $\operatorname{Gen} \mathbf{E x}(\mathbf{M}) \subseteq \mathbf{E x}\left(\mathbf{M}^{\prime}\right)$.

Let $P(p, x, j, t)=\left[\Phi_{p}(j) \leq t \wedge \Phi_{\varphi_{p}(j)}(x) \leq t\right]$. Note that, for all $p, x, j, \quad(\exists t)[P(p, x, j, t)] \Leftrightarrow(\forall$ $t)[P(p, x, j, t)] \Leftrightarrow \varphi_{\varphi_{p}(j)}(x) \downarrow$.

By the Kleene's s-m-n theorem there exists a computable $g$ such that

$$
\varphi_{g(p, j)}(x)= \begin{cases}\varphi_{\varphi_{p}\left(k^{\prime}\right)}(x), & \text { if }(\exists k \geq j)(\exists t)[P(p, x, k, t)] \wedge \\ & \left(\exists t^{\prime}\right)\left[\left\langle k^{\prime}, t^{\prime}\right\rangle=\min (\{\langle k, t\rangle \mid k \geq j \wedge P(p, x, k, t)\})\right] ; \\ \uparrow, & \text { otherwise. }\end{cases}
$$

Note that if $p, j$ and $f$ are such that

$[p$ is a 0 -generator for $f]$ and $\left[(\forall k \geq j)\left[\operatorname{card}\left(\left\{x \mid \varphi_{\varphi_{p}(k)}(x) \downarrow \neq f(x)\right\}\right)=0\right]\right]$,

then $\varphi_{g(p, j)}=f$.

Let $j_{n}^{f}=1+\max \left(\left\{j \leq n \mid(\exists y<n)\left[\right.\right.\right.$ For $\left.\left.\left.p=\mathbf{M}(f[n]), P(p, y, j, n) \wedge \varphi_{\varphi_{p}(j)}(y) \neq f(y)\right]\right\}\right)$. Note that if $f \in \operatorname{GenEx}(\mathbf{M})$, and $p=\mathbf{M}(f)$, then for all but finitely many $n, j_{n}^{f}=1+\max \left(\left\{j \mid(\exists y)\left[\varphi_{\varphi_{p}(j)}(y) \downarrow \neq f(y)\right]\right\}\right)$.

Let $\mathbf{M}^{\prime}(f[n])=g\left(\mathbf{M}(f[n]), j_{n}^{f}\right)$.

It is easy to see, using the property of $g$ discussed above, that $\operatorname{Gen} \operatorname{Ex}(\mathbf{M}) \subseteq \mathbf{E x}\left(\mathbf{M}^{\prime}\right)$.

(d) Let $\operatorname{prog}(f[n], t)=\min \left(\{n\} \cup\left\{i \leq n \mid(\forall x<n)\left[\Phi_{i}(x) \leq t \wedge \varphi_{i}(x)=f(x)\right]\right\}\right)$. 
It is easy to see that, for all $f \in \mathcal{R}$,

$$
(\forall n>\operatorname{Min} \operatorname{Prog}(f))(\stackrel{\infty}{\forall} t)[\operatorname{prog}(f[n], t)=\operatorname{MinProg}(f)]
$$

Let $\mathbf{M}$ be such that, for all $f, n, \mathbf{M}(f[n])$ is a program for $\lambda t$. $[\operatorname{prog}(f[n], t)]$.

Using (1) it immediately follows that $\mathcal{R} \in \operatorname{GenBc}(\mathbf{M})$.

Theorem 16 below shows that it may not always be possible to tradeoff anomalies by allowing learning machines to output higher order programs in the limit.

Theorem $16(\forall i)\left[\mathbf{E x}^{i+1}-\mathbf{G e n E x}^{i} \neq \emptyset\right]$.

Proof. Let $\mathcal{C}=\left\{f \mid \varphi_{f(0)}={ }^{i+1} f\right\}$. Clearly, $\mathcal{C}$ is in $\mathbf{E} \mathbf{x}^{i+1} . \mathcal{C} \notin \mathbf{G e n E x ^ { i }}$ can be shown by a simple modification of the proof that $\mathcal{C} \notin \mathbf{E x} \mathbf{x}^{i}$ in [CS83]. We omit the details.

Similarly it can be shown that,

Theorem $17 \mathbf{E x}^{*}-\bigcup_{i \in N} \operatorname{GenEx}^{i} \neq \emptyset$.

As a corollary to Theorems 16 and 17 we have

Corollary $18(\forall i)\left[\operatorname{GenEx}^{i+1}-\operatorname{GenEx}^{i} \neq \emptyset\right]$.

Corollary $19\left[\operatorname{GenEx}^{0} \subset \operatorname{GenEx}^{1} \subset \cdots \subset \operatorname{GenEx}^{i} \subset \operatorname{GenEx}^{i+1} \subset \cdots \subset\right.$ GenEx$\left.^{*}\right]$.

Theorem $20 \mathrm{Bc}-$ GenEx $^{*} \neq \emptyset$.

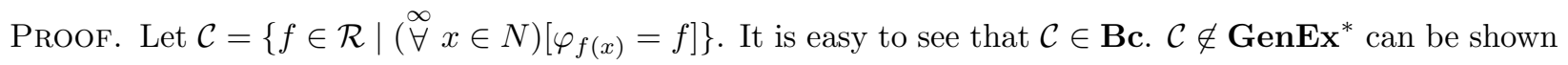
by a simple modification of the proof that $\mathcal{C} \notin \mathbf{E x}{ }^{*}$ in [CS83]. We omit the details.

Theorem $21(\forall i)\left[\operatorname{GenEx}^{i+1}-\mathbf{B c}^{i} \neq \emptyset\right]$.

Proof.

Let $\mathcal{C}=\{f \in \mathcal{R} \mid f(0)$ is an $(i+1)$-generator for $f\}$.

It is easy to see that $\mathcal{C} \in \mathbf{G e n E x ^ { i + 1 }}$. Suppose by way of contradiction that $\mathbf{M} \mathbf{B} \mathbf{c}^{i}$-identifies $\mathcal{C}$. Then, by the operator recursion theorem [Cas74], there exists an 1-1, computable function $q$ such that the (partial) functions $\varphi_{q(\cdot)}$ may be defined as follows. 
Let $x_{s}^{0}$ denote the least $x$ such that $\varphi_{q(0)}(x)$ has not been defined before stage $s$. Let $x_{s}^{1}$ denote the least $x$ such that $\varphi_{q(1)}(x)$ has not been defined before stage $s$. Let $\varphi_{q(0)}(0)=q(1)$. Let $\varphi_{q(1)}(0)=q(0)$. Go to stage 2 .

\section{Begin stage $s$}

1. For $x<x_{s}^{1}$, let $\varphi_{q(s)}(x)=\varphi_{q(1)}(x)$.

2. Let $y_{s}^{0}=x_{s}^{0}, y_{s}^{1}=x_{s}^{1}$.

\section{repeat}

2.1 Let $\varphi_{q(0)}\left(y_{s}^{0}\right)=q(s)$.

$2.2 \quad$ Let $\varphi_{q(s)}\left(y_{s}^{1}\right)=0$.

2.3 Let $y_{s}^{0}=y_{s}^{0}+1$.

$2.4 \quad$ Let $y_{s}^{1}=y_{s}^{1}+1$.

2.5 If $\left(\exists m, z \mid x_{s}^{1} \leq m \leq z<y_{s}^{1}\right)$, such that, for each $x \leq i, \Phi_{\mathbf{M}\left(\varphi_{q(s)}[m]\right)}(z+x) \leq y_{s}^{1}$ and $\varphi_{\mathbf{M}\left(\varphi_{q(s)}[m]\right)}(z+$ $x)=0$, then go to step 3 .

\section{forever}

3. Let $z$ be as found in step 2.5 .

3.1 For each $x \in\left\{w \mid x_{s}^{1} \leq w<y_{s}^{1} \wedge w \notin\{z+r \mid r \leq i\}\right\}$, let $\varphi_{q(1)}(x)=0$.

3.2 For each $x \in\{z+r \mid r \leq i\}$, let $\varphi_{q(1)}(x)=1$.

4. For $x \geq y_{s}^{1}$, whenever $\varphi_{q(1)}(x)$ gets defined, let $\varphi_{q(s)}(x)=\varphi_{q(1)}(x)$ (i.e. $\varphi_{q(s)}$ "follows" $\varphi_{q(1)}$ from now on).

(Note that this step ensures that $\varphi_{q(s)}={ }^{i+1} \varphi_{q(1)}$.)

5. Go to stage $s+1$.

End stage $s$

Now consider the following cases:

Case 1: Infinitely many stages are executed.

In this case, let $f=\varphi_{q(1)}$. Clearly, $\varphi_{q(1)} \in \mathcal{C}\left(\right.$ since $\varphi_{q(0)}$ is an $(i+1)$ generator for $\left.\varphi_{q(1)}\right)$. However, because of the success of the condition in step 2.5 in each stage, and the diagonalization in step 3.2 , for infinitely many $m, \varphi_{\mathbf{M}\left(\varphi_{q(1)}[m]\right)} \neq^{i} \varphi_{q(1)}$. Thus $f \notin \mathbf{B c}^{i}(\mathbf{M})$. 
Case 2: Stage $s$ starts but never finishes.

In this case, let $f=\varphi_{q(s)}$. Clearly, $f \in \mathcal{C}$. Moreover, for all but finitely many $m$, for infinitely many $x, \varphi_{\mathbf{M}(f[m])}(x) \neq 0$ (otherwise the test at step 2.5 would succeed). Thus $f \notin \mathbf{B c}^{i}(\mathbf{M})$.

From the above cases it follows that $\mathcal{C} \notin \mathbf{B} \mathbf{c}^{i}$.

As a corollary to Theorem 21 and Theorem 3(c) we have

Corollary $22(\forall i \geq 1)\left[\mathbf{G e n E x}^{i}-\mathbf{E x}^{*} \neq \emptyset\right]$.

The following theorem follows as a corollary to Theorems 13(b) and 15(b).

Theorem $23(\forall i)\left[\operatorname{LimEx}-\operatorname{GenEx}^{i} \neq \emptyset\right]$.

The following theorems show some tradeoff results in learning generators vis-à-vis learning Lim-programs. Note that there is a gap between the diagonalization in Theorem 24 and simulation in Theorem 25 . We leave it as an open question to find an exact tradeoff relationship between the different GenEx and LimEx learning criteria.

Theorem $24(\forall i \geq 1)\left[\operatorname{GenEx}^{i}-\operatorname{LimEx}^{\lfloor 3 i / 2\rfloor-1} \neq \emptyset\right]$

The proof of this theorem is complicated and uses the operator recursion theorem [Cas74].

Proof.

Let $\mathcal{C}=\{f \in \mathcal{R} \mid f(0)$ is an $i$-generator for $f\}$.

It is easy to see that $\mathcal{C} \in \mathbf{G e n E x} \mathbf{x}^{i}$. Suppose by way of contradiction that $\mathbf{M} \mathbf{L i m E x ^ { \lfloor 3 i / 2 \rfloor - 1 }}$-identifies $\mathcal{C}$. Then, by the operator recursion theorem [Cas74], there exists an 1-1, computable function $q$ such that the (partial) functions $\varphi_{q(\cdot)}$ may be described as follows.

By the Kleene s-m-n theorem, there exists a computable $L$ such that for all $p, x, t$,

$$
\varphi_{L(p)}(x, t)= \begin{cases}\varphi_{p}\left(x, t^{\prime}\right), & \left(\exists t^{\prime \prime} \leq t\right)\left[\Phi_{p}\left(x, t^{\prime \prime}\right) \leq t\right] \wedge \\ & t^{\prime}=\max \left(\left\{t^{\prime \prime} \leq t \mid \Phi_{p}\left(x, t^{\prime \prime}\right) \leq t\right\}\right) ; \\ 0, & \text { otherwise. }\end{cases}
$$

Note that, for all $p, \varphi_{L(p)}$ is a total function, and $\varphi_{p}^{\star} \subseteq \varphi_{L(p)}^{\star}$.

Let $x_{s}^{0}$ denote the least $x$, such that $\varphi_{q(0)}(x)$ has not been defined before stage $s$. Let $x_{s}^{1}$ denote the least $x$, such that $\varphi_{q(1)}(x)$ has not been defined before stage $s$.

Let $\varphi_{q(0)}(0)=q(1)$. Let $\varphi_{q(1)}(0)=q(0)$. Go to stage 1 . 


\section{Begin stage $s$}

1. For $x<x_{s}^{1}$, let $\varphi_{q(2 s)}(x)=\varphi_{q(2 s+1)}(x)=\varphi_{q(1)}(x)$.

For all $z<i$, let $\varphi_{q(2 s)}\left(x_{s}^{1}+z\right)=0$ and $\varphi_{q(2 s+1)}\left(x_{s}^{1}+z\right)=1$.

2. For $b_{0}, b_{1}, \ldots, b_{i-1} \in\{0,1\}$ and $y \in N$, define $f_{b_{0} b_{1} \ldots b_{i-1}, y}$ as follows:

$$
f_{b_{0} b_{1} \ldots b_{i-1}, y}(x)= \begin{cases}\varphi_{q(1)}(x), & \text { if } x<x_{s}^{1} \\ b_{x-x_{s}^{1}}, & \text { if } x_{s}^{1} \leq x \leq x_{s}^{1}+i-1 ; \\ y, & \text { if } x_{s}^{1}+i \leq x<x_{s}^{1}+\left\lfloor\frac{3 i}{2}\right\rfloor \\ 0, & \text { otherwise. }\end{cases}
$$

3. Let $y_{s}^{0}=x_{s}^{0}$ and $y_{s}^{1}=x_{s}^{1}+\left\lfloor\frac{3 i}{2}\right\rfloor$.

\section{repeat}

3.1 Let $\varphi_{q(2 s)}\left(y_{s}^{1}\right)=\varphi_{q(2 s+1)}\left(y_{s}^{1}\right)=0$.

3.2 Let $C_{1}=\operatorname{card}\left(\left\{x \mid x_{s}^{1} \leq x<x_{s}^{1}+i \wedge \varphi_{L\left(\mathbf{M}\left(\varphi_{q(1)}\left[x_{s}^{1}\right]\right)\right)}\left(x, y_{s}^{1}\right)=1\right\}\right)$.

3.3 if $C_{1} \geq\left\lceil\frac{i}{2}\right\rceil$ then

$$
\text { let } \varphi_{q(0)}\left(y_{s}^{0}\right)=q(2 s) \text {. }
$$

else

$$
\text { let } \varphi_{q(0)}\left(y_{s}^{0}\right)=q(2 s+1) .
$$

endif

$3.4 y_{s}^{0}=y_{s}^{0}+1, y_{s}^{1}=y_{s}^{1}+1$.

3.5 If there exist $b_{0}, b_{1}, \ldots, b_{i-1} \in\{0,1\}$ and $y \leq y_{s}^{1}$ such that $\mathbf{M}\left(\varphi_{q(1)}\left[x_{s}^{1}\right]\right) \neq \mathbf{M}\left(f_{b_{0} b_{1} \ldots b_{i-1}, y}\left[y_{s}^{1}\right]\right)$, then go to step 4 .

\section{forever}

4. Let $b_{0}, b_{1}, \ldots, b_{i-1}, y$ be as found in step 3.5.

4.1 For $z<i$, let $\varphi_{q(1)}\left(x_{s}^{1}+z\right)=b_{z}$.

4.2 For $z<\left\lfloor\frac{i}{2}\right\rfloor$, let $\varphi_{q(2 s)}\left(x_{s}^{1}+i+z\right)=\varphi_{q(2 s+1)}\left(x_{s}^{1}+i+z\right)=\varphi_{q(1)}\left(x_{s}^{1}+i+z\right)=y$.

4.3 For $z$ such that $x_{s}^{1}+\left\lfloor\frac{3 i}{2}\right\rfloor \leq z<y_{s}^{1}$, let $\varphi_{q(1)}\left(x_{s}^{1}+i+z\right)=0$.

4.4 For $x \geq y_{s}^{1}$, whenever $\varphi_{q(1)}(x)$ gets defined, let $\varphi_{q(2 s)}(x)=\varphi_{q(2 s+1)}(x)=\varphi_{q(1)}(x)\left(\right.$ i.e. $\varphi_{q(2 s)}$ and $\varphi_{q(2 s+1)}(x)$ "follow" $\varphi_{q(1)}$ from now on; also, because of step $4, \varphi_{q(2 s)}={ }^{i} \varphi_{q(1)}$ and $\left.\varphi_{q(2 s+1)}={ }^{i} \varphi_{q(1)}\right)$. 
5. Go to stage $s+1$.

End stage $s$

Now consider the following cases.

Case 1: Infinitely many stages are executed.

In this case, let $f=\varphi_{q(1)}$. Clearly, $f \in \mathcal{C}$ (since $\varphi_{q(0)}$ is a $i$ generator for $\left.f\right)$. However, $\mathbf{M}(f) \uparrow$ (since, the only way infinitely many stages can be executed is the success of the condition at step 3.5 in each stage $s)$. Thus $f \notin \mathbf{L i m E x ^ { \lfloor 3 i / 2 \rfloor - 1 }}$.

Case 2: Stage $s$ starts but never finishes.

For all $b_{0}, b_{1}, \ldots, b_{i-1} \in\{0,1\}$ and $y \in N$, let $f_{b_{0} b_{1} \ldots b_{i-1}, y}$ be as defined in step 2 of stage $s$. Note that in this case, for all $b_{0}, b_{1}, \ldots, b_{i-1} \in\{0,1\}$ and $y \in N, \mathbf{M}\left(f_{b_{0} b_{1} \ldots b_{i-1}, y}\right)=\mathbf{M}\left(\varphi_{q(1)}\left[x_{s}^{1}\right]\right)$.

Let $\operatorname{Conv}_{1}=\left\{x \mid\left[x_{s}^{1} \leq x<x_{s}^{1}+i\right] \bigwedge\left[\varphi_{\mathbf{M}\left(\varphi_{q(1)}\left[x_{s}^{1}\right]\right)}^{\star}(x) \downarrow=1\right]\right\}$.

Let Convnon $_{1}=\left\{x \mid\left[x_{s}^{1} \leq x<x_{s}^{1}+i\right] \bigwedge\left[\varphi_{\mathbf{M}\left(\varphi_{q(1)}^{\star}\left[x_{s}^{1}\right]\right)}(x) \downarrow \neq 1\right]\right\}$.

Let $y$ be such that, for all $z \in\left\{z^{\prime} \mid x_{s}^{1}+i \leq z^{\prime}<x_{s}^{1}+\left\lfloor\frac{3 i}{2}\right\rfloor\right\}, \varphi_{\mathbf{M}\left(\varphi_{q(1)}\left[x_{s}^{1}\right]\right)}(z) \neq y$ (note that there exists such a $y$ ).

Case 2.1: $\operatorname{card}\left(\right.$ Conv $\left._{1}\right) \geq\lceil i / 2\rceil$

Note that in this case $(\stackrel{\infty}{\forall} x)\left[\varphi_{q(0)}(x)=q(2 s)\right]$. For $k<i$, if $x_{s}^{1}+k \in$ Conv $_{1}$, then let $b_{k}=0$; else, let $b_{k}=1$. Note that $f_{b_{0} b_{1} \ldots b_{i-1}, y} \neq^{\lfloor 3 i / 2\rfloor-1} \varphi_{\mathbf{M}\left(\varphi_{q(1)}\left[x_{s}^{1}\right]\right)}$. Since $\left(i-\operatorname{card}\left(\operatorname{Conv}_{1}\right)\right)+\left\lfloor\frac{i}{2}\right\rfloor \leq i$, $f_{b_{0} b_{1} \ldots b_{i-1}, y}={ }^{i} \varphi_{q(2 s)}$. Thus $f_{b_{0} b_{1} \ldots b_{i-1}, y} \in \mathcal{C}-\mathbf{L i m E x}^{\lfloor 3 i / 2\rfloor-1}(\mathbf{M})$.

Case 2.2: $\operatorname{card}\left(\right.$ Convnon $\left._{1}\right) \geq\lceil i / 2\rceil$.

Note that in this case $(\stackrel{\infty}{\forall} x)\left[\varphi_{q(0)}(x)=q(2 s+1)\right]$. For $k<i$, if $x_{s}^{1}+k \in$ Convnon $_{1}$, then let $b_{k}=1$; else, let $b_{k}=0$. Note that $f_{b_{0} b_{1} \ldots b_{i-1}, y} \neq^{\lfloor 3 i / 2\rfloor-1} \varphi_{\mathbf{M}\left(\varphi_{q(1)}\left[x_{s}^{1}\right]\right)}^{\star}$. Since $(i-$ $\operatorname{card}\left(\right.$ Convnon $\left.\left._{1}\right)\right)+\left\lfloor\frac{i}{2}\right\rfloor \leq i, f_{b_{0} b_{1} \ldots b_{i-1}, y}={ }^{i} \varphi_{q(2 s+1)}$. Thus $f_{b_{0} b_{1} \ldots b_{i-1}, y} \in \mathcal{C}-\mathbf{L i m E x}^{\lfloor 3 i / 2\rfloor-1}(\mathbf{M})$.

Case 2.3: $\operatorname{card}\left(\right.$ Conv $\left._{1}\right)<\lceil i / 2\rceil$ and $\operatorname{card}\left(\right.$ Convnon $\left._{1}\right)<\lceil i / 2\rceil$.

Let $S \subseteq\left\{x_{s}^{1}+z \mid z<i\right\}$ be a set of cardinality $\lceil i / 2\rceil$ such that Convnon $_{1} \subseteq S$ and $S \cap \operatorname{Conv}_{1}=\emptyset$ (note that there exists such an $S$ ). Note that in this case $(\forall x)\left[\varphi_{q(0)}(x) \in\right.$ $\{q(2 s), q(2 s+1)\}]$. For $k<i$, if $x_{s}^{1}+k \in S$, then let $b_{k}=1$; else, let $b_{k}=0$. Note that 


$$
\begin{aligned}
& f_{b_{0} b_{1} \ldots b_{i-1}, y} \neq{ }^{\lfloor 3 i / 2\rfloor-1} \varphi_{\mathbf{M}\left(\varphi_{q(1)}\left[x_{s}^{1}\right]\right)}^{\star} \text {. Since } \operatorname{card}(S)+\left\lfloor\frac{i}{2}\right\rfloor \leq i, f_{b_{0} b_{1} \ldots b_{i-1}, y}={ }^{i} \varphi_{q(2 s)} \text {. Also, since } \\
& (i-\operatorname{card}(S))+\left\lfloor\frac{i}{2}\right\rfloor \leq i, f_{b_{0} b_{1} \ldots b_{i-1}, y}={ }^{i} \varphi_{q(2 s+1)} . \text { Thus } f_{b_{0} b_{1} \ldots b_{i-1}, y} \in \mathcal{C}-\mathbf{L i m E x} \mathbf{x}^{\lfloor 3 i / 2\rfloor-1}(\mathbf{M}) .
\end{aligned}
$$

From the above cases it follows that $\mathcal{C} \notin \mathbf{L i m E x} \mathbf{x}^{\lfloor 3 i / 2\rfloor-1}$.

\section{Theorem $25(\forall i)\left[\operatorname{GenEx}^{i} \subset \operatorname{LimEx}^{2 i}\right]$.}

Proof. We show that $\mathbf{G e n E x}^{i} \subseteq \mathbf{L i m E x ^ { 2 i }}$. Proper containment will then follow using Theorem 23.

Let $F$ be the (partial) function from triplets of natural numbers to sequences defined as follows.

$F(p, r, m)=$ the lexicographically least sequence $\sigma$ of length $m+1$, if such a sequence exists, which satisfies (2).

$$
(\forall j \mid r \leq j<m)\left[\operatorname{card}\left(\left\{x \mid x \leq m \wedge \Phi_{p}(j) \leq m \wedge \Phi_{\varphi_{p}(j)}(x) \leq m \wedge \varphi_{\varphi_{p}(j)}(x) \neq \sigma(x)\right\}\right) \leq i\right] ;
$$

$F(p, r, m)=\uparrow$ if no $\sigma$ of length $m+1$ satisfies (2).

By the Kleene's s-m-n theorem [Rog67] there exists a computable $g$ such that for all $p, x, r, t$

$$
\varphi_{g(p, r)}(x, t)=(F(p, r, t+x))(x) .
$$

Let $\operatorname{Err}(f, n, p)=\max \left(\left\{j+1 \mid \operatorname{card}\left(\left\{x<n \mid \Phi_{p}(j) \leq n \wedge \Phi_{\varphi_{p}(j)}(x) \leq n \wedge \varphi_{\varphi_{p}(j)}(x) \neq f(x)\right\}\right)>i\right\}\right)$.

Given $\mathbf{M}$, define $\mathbf{M}^{\prime}$ as follows. $\mathbf{M}^{\prime}(f[n])=g(\mathbf{M}(f[n]), \operatorname{Err}(f, n, \mathbf{M}(f[n])))$.

It is easy to see that $\operatorname{GenEx}^{i}(\mathbf{M}) \subseteq \operatorname{LimEx}^{2 i}\left(\mathbf{M}^{\prime}\right)$.

\section{Theorem 26 LimEx$^{*} \subseteq$ GenEx*}

Proof. We will describe a computable function $g$ such that, for all $p, g(p)$ is such that

$$
\varphi_{g(p)} \text { is total and }(\forall f \in \mathcal{R})\left[\varphi_{p}^{\star}={ }^{*} f \Rightarrow g(p) \text { is a } * \text {-generator for } f\right] .
$$

Given, $\mathbf{M}$ define $\mathbf{M}^{\prime}(f[n])=g(\mathbf{M}(f[n]))$. It is easy to see that $\mathbf{L i m E x} \mathbf{x}^{*}(\mathbf{M}) \subseteq \mathbf{G e n E x}^{*}\left(\mathbf{M}^{\prime}\right)$.

Now we show how to obtain the $g$ as claimed above.

By Kleene's s-m-n theorem there exist computable $h, \operatorname{prog}, g$ such that the following three conditions hold. 
(a) For all $p, x, t$,

$$
\varphi_{h(p)}(x, t)= \begin{cases}\varphi_{p}\left(x, t^{\prime}\right), & \left(\exists t^{\prime \prime} \leq t\right)\left[\Phi_{p}\left(x, t^{\prime \prime}\right) \leq t\right] \wedge \\ & t^{\prime}=\max \left(\left\{t^{\prime \prime} \leq t \mid \Phi_{p}\left(x, t^{\prime \prime}\right) \leq t\right\}\right) ; \\ 0, & \text { otherwise. }\end{cases}
$$

Note that for all $p, \varphi_{h(p)}$ is a total function. Moreover, $\varphi_{p}^{\star} \subseteq \varphi_{h(p)}^{\star}$.

(b) For all $p, j, y$,

$$
\operatorname{prog}(p, j, y)=\min \left(\{j\} \cup\left\{i<j \mid \operatorname{card}\left(\left\{x \leq j \mid \Phi_{i}(x)>y \vee \varphi_{i}(x) \neq \varphi_{h(p)}(x, y)\right\}\right) \leq i\right\}\right) .
$$

(c) For all $p, j, y,\left[\varphi_{g(p)}\right.$ is total $\left.\wedge \varphi_{\varphi_{g(p)}(j)}(y)=\varphi_{\operatorname{prog}(p, j, y)}(y)\right]$.

Suppose, $p$ is such that for some $f \in \mathcal{R}, \varphi_{p}^{\star}={ }^{*} f$. Let finerr $(p)=\min \left(\left\{i \mid \operatorname{card}\left(\left\{x \mid \varphi_{p}^{\star}(x) \uparrow \vee \varphi_{i}(x) \neq\right.\right.\right.\right.$ $\left.\left.\left.\left.\varphi_{p}^{\star}(x)\right\}\right) \leq i\right\}\right)$. It is easy to see that $\varphi_{\text {finerr }(p)}={ }^{*} f$. Moreover,

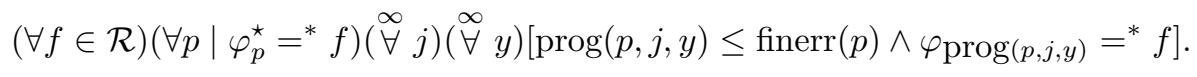

It follows that,

$$
(\forall f \in \mathcal{R})\left(\forall p \mid \varphi_{p}^{\star}=^{*} f\right)(\stackrel{\infty}{\forall} j)(\stackrel{\infty}{\forall} y)\left[\varphi_{\operatorname{prog}(p, j, y)}(y)=f(y)\right] .
$$

It follows from (7) and the definition of $g$, that, $g$ satisfies (4).

\section{Open Problems}

Note that there is a gap between the diagonalization in Theorem 24 and simulation in Theorem 25 . We leave it as an open question to find an exact tradeoff relationship between the different GenEx and LimEx learning criteria. It is also open at present whether $\mathbf{G e n E x}^{*}=\mathbf{L i m E x}^{*}$ (note that by Theorem $26 \mathbf{L i m E x}^{*} \subseteq$ GenEx*).

\section{References}

[AS83] D. Angluin and C. Smith. A survey of inductive inference: Theory and methods. Computing Surveys, 15:237-289, 1983. 
[Bar74] J. M. Barzdin. Two theorems on the limiting synthesis of functions. In Theory of Algorithms and Programs, Latvian State University, Riga, 210:82-88, 1974. In Russian.

[BB75] L. Blum and M. Blum. Toward a mathematical theory of inductive inference. Information and Control, 28:125-155, 1975.

[Blu67] M. Blum. A machine independent theory of the complexity of recursive functions. Journal of the ACM, 14:322-336, 1967.

[Cas74] J. Case. Periodicity in generations of automata. Mathematical Systems Theory, 8:15-32, 1974.

[Cas86] J. Case. Learning machines. In W. Demopoulos and A. Marras, editors, Language Learning and Concept Acquisition. Ablex Publishing Company, 1986.

[Che81] K. Chen. Tradeoffs in Machine Inductive Inference. PhD thesis, SUNY at Buffalo, 1981.

[CJS92] J. Case, S. Jain, and A. Sharma. On learning limiting programs. International Journal of Foundations of Computer Science, 3(1):93-115, 1992.

[CL82] J. Case and C. Lynes. Machine inductive inference and language identification. In M. Nielsen and E. M. Schmidt, editors, Proceedings of the 9th International Colloquium on Automata, Languages and Programming, volume 140, pages 107-115. Springer-Verlag, Berlin, 1982.

[Cra53] W. Craig. On axiomatizability within a system. Journal of Symbolic Logic, 18:30-32, 1953.

[CS83] J. Case and C. Smith. Comparison of identification criteria for machine inductive inference. Theoretical Computer Science, 25:193-220, 1983.

[Fef60] S. Feferman. Arithmetization of metamathematics in a general setting. Fundamenta Mathematicae, 49:35-92, 1960 .

[Fis65] P. Fischer. Theory of provable recursive functions. Transactions of the American Mathematical Society, 117:494-520, 1965.

[Ful85] M. Fulk. A Study of Inductive Inference machines. PhD thesis, SUNY at Buffalo, 1985.

[Gol65] E. M. Gold. Limiting recursion. Journal of Symbolic Logic, 30:28-48, 1965. 
[Gol67] E. M. Gold. Language identification in the limit. Information and Control, 10:447-474, 1967.

[HU79] J. Hopcroft and J. Ullman. Introduction to Automata Theory Languages and Computation. Addison-Wesley Publishing Company, 1979.

[Kre51] G. Kreisel. On the interpretation of non-finitist proofs. Journal of Symbolic Logic, 17:241-267, 1951.

[Kre58] G. Kreisel. Mathematical significance of consistency proofs. Journal of Symbolic Logic, 23:155-182, 1958.

[KW80] R. Klette and R. Wiehagen. Research in the theory of inductive inference by GDR mathematicians - A survey. Information Sciences, 22:149-169, 1980.

[LMF76] N. Lynch, A. Meyer, and M. Fischer. Relativization of the theory of computational complexity. Transactions of the American Mathematical Society, 220:243-287, 1976.

[Men86] E. Mendelson. Introduction to Mathematical Logic. Brooks-Cole, San Francisco, 1986. 3rd Edition.

[MY78] M. Machtey and P. Young. An Introduction to the General Theory of Algorithms. North Holland, New York, 1978.

[OSW86] D. Osherson, M. Stob, and S. Weinstein. Systems that Learn, An Introduction to Learning Theory for Cognitive and Computer Scientists. MIT Press, Cambridge, Mass., 1986.

[Put65] H. Putnam. Trial and error predicates and the solution to a problem of Mostowski. Journal of Symbolic Logic, 30:49-57, 1965.

[RC92] J. Royer and J. Case. Intensional Subrecursion and Complexity Theory. Research Notes in Theoretical Science. Pitman Press, being revised for expected publication, 1992.

[Rog57] H. Rogers. Provably recursive functions. Bulletin of the American Mathematical Society, 63:140, 1957.

[Rog58] H. Rogers. Gödel numberings of partial recursive functions. Journal of Symbolic Logic, 23:331-341, 1958. 
[Rog67] H. Rogers. Theory of Recursive Functions and Effective Computability. McGraw Hill, New York, 1967. Reprinted, MIT Press 1987.

[Ros84] H. Rose. Subrecursion: Functions and Hierarchies. Oxford University Press, 1984.

[Sha71] N. Shapiro. Review of "Limiting recursion" by E.M. Gold and "Trial and error predicates and the solution to a problem of Mostowski" by H. Putnam. Journal of Symbolic Logic, 36:342, 1971.

[Sho59] J. Shoenfield. On degrees of unsolvability. Annals of Mathematics, 69:644-653, 1959.

[Sho71] J. Shoenfield. Degrees of Unsolvability. North-Holland, 1971.

[Soa87] R. Soare. Recursively Enumerable Sets and Degrees. Springer-Verlag, 1987.

[Wie78] R. Wiehagen. Zur Theorie der Algorithmischen Erkennung. PhD thesis, Humboldt-Universitat, Berlin, 1978. 